\title{
Perpendicular magnetic anisotropy in Nd-Co alloy films nanostructured by di-block copolymer templates
}

\author{
F. Valdés-Bango, ${ }^{1,2}$ F. J. García Alonso, ${ }^{2,3}$ G. Rodríguez-Rodríguez, ${ }^{1,2}$ \\ L. Morán Fernandez, ${ }^{3}$ A. Anillo, ${ }^{3}$ L. Ruiz-Valdepeñas, ${ }^{4}$ E. Navarro, ${ }^{4}$ J. L. Vicent, ${ }^{4,5}$ \\ M. Vélez, ${ }^{1}$ J. I. Martín, ${ }^{1,2}$ and J. M. Alameda ${ }^{1,2}$ \\ ${ }^{1}$ Depto. Física, Fac. Ciencias, Universidad de Oviedo, Av. Calvo Sotelo s/n, 33007 Oviedo, Spain \\ ${ }^{2}$ CINN (CSIC-Universidad de Oviedo-Principado de Asturias) Llanera, Spain \\ ${ }^{3}$ Depto. Química Orgánica e Inorgánica, Universidad de Oviedo, 33007 Oviedo, Spain \\ ${ }^{4}$ Dpto. Física de Materiales, Universidad Complutense, 28040 Madrid, Spain \\ ${ }^{5}$ IMDEA-Nanociencia, Cantoblanco, 28049 Madrid, Spain
}

(Received 31 July 2012; accepted 25 September 2012; published online 19 October 2012)

\begin{abstract}
Nd-Co amorphous magnetic films with perpendicular magnetic anisotropy have been grown on nanostructured templates prepared with self-organized di-block poly(styrene)-blockpoly(4-vinylpyridine) copolymer layers with a periodic structure of $60 \mathrm{~nm}$ spaced pores. These templates modify both the magnetic film topography and mechanical strain on a local scale. The effect of these structural changes is particularly noticeable in the low thickness range of the magnetic films where the transition from in-plane to out-of plane magnetization takes place. The Nd-Co films grown on the copolymer template present lower perpendicular magnetic anisotropy and, also, stronger stripe domain pinning effects in comparison with reference films grown on flat Si substrates. (C) 2012 American Institute of Physics.
\end{abstract}

[http://dx.doi.org/10.1063/1.4759276]

\section{INTRODUCTION}

Rare earth-transition metal (RE-TM) amorphous alloys have long been the subject of active research due to their interesting magnetic properties for a wide range of applications and the possibility to control them through composition and preparation conditions. ${ }^{1}$ In many cases, these RE-TM alloy films display perpendicular magnetic anisotropy (PMA) that is of use for magneto-optical recording. ${ }^{2}$ Recently, the need to control the magnetic properties at the nanoscale has led to the development of different methods to modify PMA materials on a local scale. ${ }^{3-16}$ One approach is to locally modify chemical order by ion irradiation of the PMA films either through lithography fabricated masks ${ }^{3,4}$ or by focused ion beams, 5,6 which has the advantage that the final film topography is very planar. Another possibility is to alter film topography by the use of prepatterned substrates. 7,8 Then, "bottom-up" fabrication methods that use self-organized materials appear as a good possibility to obtain submicrometric resolution over large areas. ${ }^{9-13}$ For example, porous alumina templates have been used to fabricate PMA antidot arrays with engineered coercivity at the expense of a very rough topography. ${ }^{14-16}$ Thin films of self-organized di-block copolymers provide an alternative route to fabricate nanostructured templates for metal films, ${ }^{9-11,13,17-19}$ that have been used to control different magnetic properties such as bit size in patterned magnetic media, ${ }^{9}$ exchange bias in $\mathrm{Fe} / \mathrm{FeF}_{2}$ bilayers ${ }^{17}$ or coercivity in permalloy films, ${ }^{18}$ and spin valves. ${ }^{19}$

Most of the works on local modifications of PMA have been focused on $\mathrm{Co}(\mathrm{Pt}, \mathrm{Pd})$ and $\mathrm{Fe}(\mathrm{Pt}, \mathrm{Pd})$ alloys ${ }^{3-5,7,10,12}$ due to their strong PMA and large $\mathrm{Q}$ factors $\left(\mathrm{Q}=\mathrm{K}_{\mathrm{N}} / 2 \pi \mathrm{M}_{\mathrm{S}}{ }^{2}\right.$ with $\mathrm{K}_{\mathrm{N}}$ the perpendicular anisotropy and $\mathrm{M}_{\mathrm{S}}$ the saturation magnetization). However, there are only a few reports on locally modified RE-TM alloys ${ }^{6,11}$ in spite of their interesting magneto-optical properties. Actually, it must be noted that this kind of low-Q materials present a different regime of stripe domains (i.e., weak stripe patterns) in which the magnetization only orients out of plane above a critical thickness ${ }^{20,21}$ and, thus, there is a significant interplay between inplane and out-of-plane magnetization components that could also be tailored by the local nanostructuration processes.

In this work, we have prepared self-organized di-block copolymer layers to be used as nanostructured templates for the deposition of Nd-Co amorphous alloy films. These alloys display a weak PMA ${ }^{22,23}$ that has been explained in terms of a random magnetic anisotropy model. ${ }^{1}$ In this framework, anisotropies in the local atomic environments of the magnetic atoms account for the observed PMA, ${ }^{24,25}$ and thus it should be quite sensitive to local changes induced by a nanostructuring process. Particular attention has been paid to the effect of the copolymer templates in the low thickness regime of the Nd-Co films where the transition from in-plane to out-of-plane magnetization takes place.

\section{FABRICATION}

\section{A. Nanostructured template fabrication}

A micellar di-block copolymer film was obtained by spin-coating a solution of poly(styrene)-block-poly(4-vinylpyridine) (PS-b-P4VP) copolymer in toluene (0.5 wt. \%) onto a silicon wafer following a procedure previously described, ${ }^{26}$ with minor modifications. The poly(styrene)-block-poly(4vinylpyridine) sample was purchased from Polymer Source and was used without further purification $(\mathrm{Mn}, \mathrm{PS}=41.5 \mathrm{~kg} /$ $\mathrm{mol}, \mathrm{Mn}, \mathrm{P} 4 \mathrm{VP}=17.5 \mathrm{~kg} / \mathrm{mol}, \mathrm{Mw} / \mathrm{Mn}=1.07)$. The copolymer was dissolved in toluene at $70^{\circ} \mathrm{C}$ for $12 \mathrm{~h}$ and, after 
cooling it down to room temperature, the solution was filtered through Millipore $0.45 \mu \mathrm{m}$ Nylon filters. The spherical micelles with a P4VP core surrounded by a PS shell are already present in solution ${ }^{27}$ since toluene is a good solvent for PS but a non-solvent for P4VP.

The surface of the silicon substrates was hydroxylated by a treatment with piranha before spin-coating. The highly polished Si plates $(7 \times 7 \mathrm{~mm})$ were first cleaned in an ultrasonic bath for $15 \mathrm{~min}$ with acetone and Millipore water. Then, the wafers were treated with piranha solution $(30 \%$ $\mathrm{H}_{2} \mathrm{O}_{2} / 97 \% \mathrm{H}_{2} \mathrm{SO}_{4}=1 / 3$, v/v) for $30 \mathrm{~min}$ also in an ultrasonic bath. The surfaces were washed successively with distilled and Millipore water and then dried with nitrogen flow and later in an oven at $120^{\circ} \mathrm{C}$ for $1 \mathrm{~h}$ prior to use. Then, the micellar solution of PS-b-P4VP in toluene (0.5 wt. \%) was spin-coated at $2000 \mathrm{rpm}$ onto a hydroxylated $\mathrm{Si}(100)$ substrate and maintained overnight under static vacuum. The resulting copolymer film thickness, measured by ellipsometry, is $26 \mathrm{~nm}$. The film surface, characterized by atomic force microscopy (AFM), shows a disordered micellar structure with $60 \mathrm{~nm}$ average micelle size (see Figure 1(a)).

Then, the films were exposed to tetrahydrofuran (THF) vapor for $3 \mathrm{~h}$ in a closed vessel at $23^{\circ} \mathrm{C}$ and dried in open air for $3 \mathrm{~h}$ and under static vacuum overnight. After this process, the lateral ordering of the micelles was markedly enhanced (see Figure 1(b)) due to the fact that THF is a good solvent for both PS and P4VP.

Later, the films were immersed in absolute ethanol at room temperature for $20 \mathrm{~min}$. Then, the wafers were gently shaken to eliminate the alcohol drops and dried in open air for $3 \mathrm{~h}$ and under static vacuum overnight. The immersion of the film into ethanol, a good solvent for P4VP and a nonsolvent for PS, resulted in the generation of a highly oriented array of nanopores (see Figure 1(c)) through a core-corona inversion in the micelles. ${ }^{26}$ Average separation distance is $55-65 \mathrm{~nm}$, similar to that seen in the film before exposure to alcohol, and average pore radius is $10-20 \mathrm{~nm}$.

Finally, a $10 \mathrm{~nm}$ thick $\mathrm{Al}$ film is grown by sputtering on top of the surface reconstructed diblock copolymer film. The purpose of this metal layer is as a buffer to prevent oxidation of the Nd-Co magnetic film. Figure 1(d) is the AFM image of the nanostructured $\mathrm{Al}(10 \mathrm{~nm}) /$ copolymer/Si(100) template on which the magnetic layer will be deposited. The Al layer shows a similar periodic hexagonal geometry as the underlying self-organized copolymer film but made up of a periodic arrangement of bumps instead of small sized pores. This could be related to modifications of the polymer layer surface during the sputtering process or to a preferential doping of one of the copolymer components by the metal atoms as reported by Abul Kashem et al. ${ }^{28}$ for thin metal layers. Thus, this nanostructured $\mathrm{Al}$ surface will serve as a template for the deposition of the RE-TM alloy magnetic layers.

\section{B. Magnetic layer fabrication and characterization}

A series of amorphous Nd-Co films with thickness ( $t$ ) in the range $30-75 \mathrm{~nm}$ have been deposited on top of the nanostructured $\mathrm{Al}(10 \mathrm{~nm}) /$ copolymer/Si(100) templates in order to analyze the effect of nanostructuration as a function of sample thickness. The magnetic layers were prepared by DC sputtering at room temperature in a vacuum chamber with base pressure $4 \times 10^{-8}$ mbar and Ar pressure $3 \times 10^{-3}$ mbar during the
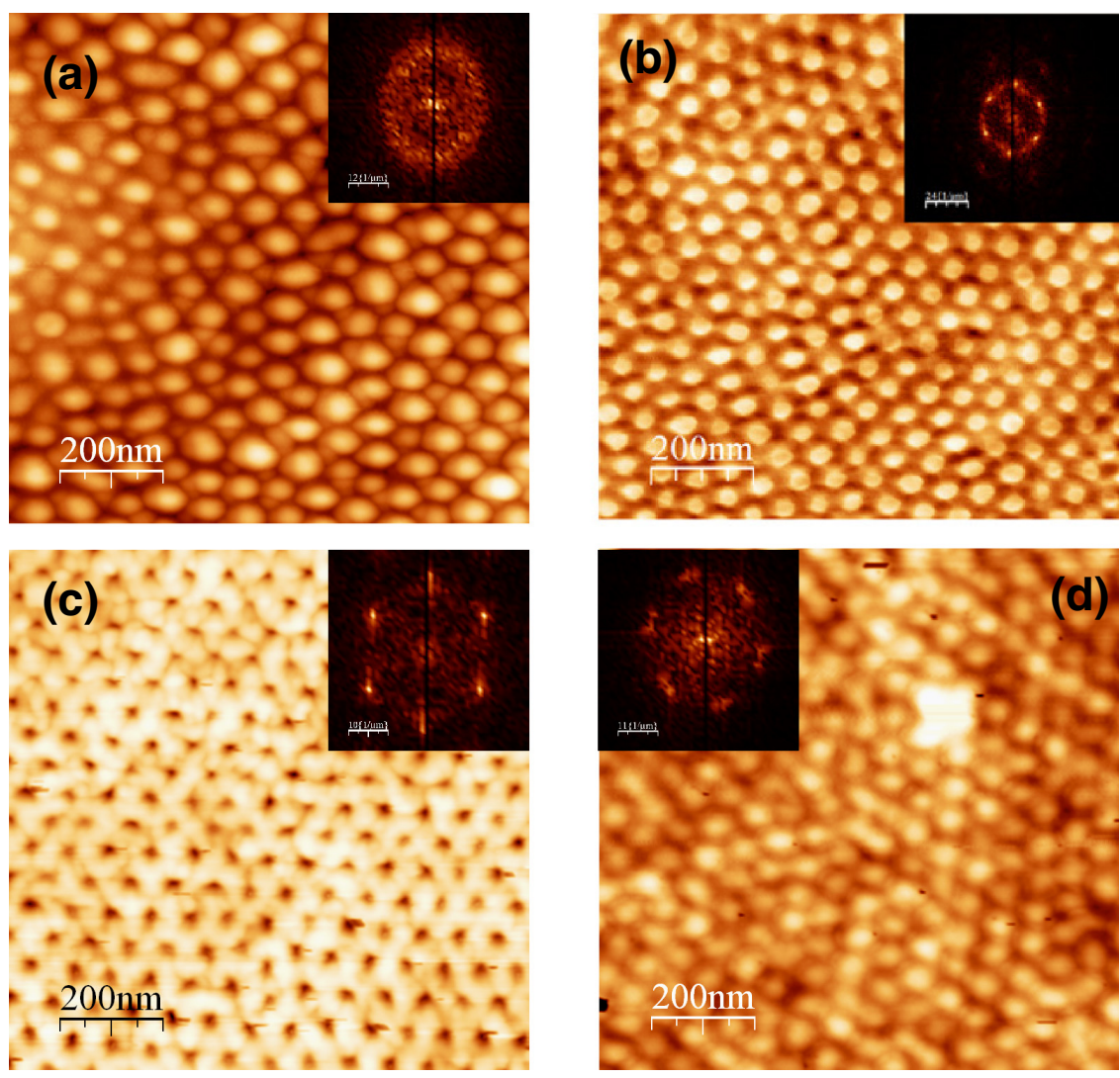

FIG. 1. AFM images of diblock copolymers (a) after spin-coating on $\mathrm{Si}$; (b) after THF ordering; (c) after ethanol exposure; (d) after deposition of $10 \mathrm{~nm}$ Al. Top insets show the corresponding FFT patterns. 
deposition process. $^{22}$ The samples were grown by cosputtering from independent pure $\mathrm{Nd}$ and Co targets. Target powers during deposition were adjusted to obtain alloy films with composition $\mathrm{NdCo}_{5}$ that, at room temperature, have saturation magnetization $\mathrm{M}_{\mathrm{S}}=1100 \mathrm{emu} / \mathrm{cm}^{3}$ and perpendicular anisotropy $\left(\mathrm{K}_{\mathrm{N}}\right)$ of the order of $10^{6} \mathrm{erg} / \mathrm{cm}^{3},{ }^{22}$ i.e., these films are characterized by a low $\mathrm{Q}$-factor, $\mathrm{Q}=\mathrm{K}_{\mathrm{N}} / 2 \pi \mathrm{M}_{\mathrm{S}}^{2} \approx 0.1$. The films also present a small in-plane uniaxial anisotropy of the order of $10^{2} \mathrm{erg} / \mathrm{cm}^{3}$ induced by deposition geometry. Finally, a $5 \mathrm{~nm}$ thick $\mathrm{Al}$ capping layer was deposited on top to avoid oxidation. A second series of $\mathrm{Al}(5 \mathrm{~nm}) / \mathrm{Nd}-\mathrm{Co}(\mathrm{t})$ films was grown simultaneously on top of flat $\mathrm{Al}(10 \mathrm{~nm}) / \mathrm{Si}(100)$ substrates for comparison.

Figure 2 shows the results of the atomic force microscopy characterization of an $\mathrm{Al}(5 \mathrm{~nm}) / \mathrm{Nd}-\mathrm{Co}(45 \mathrm{~nm}) / \mathrm{Al}(10 \mathrm{~nm}) / \mathrm{co}-$ polymer/Si(100) sample performed in the dynamic mode with a $75 \mathrm{kHz}$ resonance frequency cantilever. The height image (Fig. 2(a)) shows a relatively smooth topography with a long wavelength roughness of $1.2 \mathrm{~nm}$ rms amplitude that corresponds to the grains in the $\mathrm{Al}$ capping layer. Only a faint signature of the short wavelength corrugation due to the underlying nanostructured template can be seen in the topography image. On the other hand, the corresponding phase image (Fig. 2(b)) shows a weak but clear contrast of dots with $54 \mathrm{~nm}$ spacing, i.e., with a similar periodicity to the underlying copolymer template. This periodic phase contrast is only present in the samples grown on the copolymer templates but it is not seen on the $\mathrm{Nd}-\mathrm{Co} / \mathrm{Si}$ samples. Phase images in dynamic mode AFM operation can be interpreted as energy dissipation images due to tip-sample inelastic interactions. ${ }^{29,30}$ In the Nd-Co/template samples, the observed phase contrast is enhanced as a function of both free oscillation amplitude and setpoint used during the dynamic mode AFM measurement suggesting that it can be attributed to changes in the micromechanical properties of the samples. ${ }^{31}$ It implies that the changes induced by the copolymer template on the magnetic film will be two fold: first, there should be a topographic effect on the bottom magnetic layer surface due to the nanostructured corrugation of the $\mathrm{Al}(10 \mathrm{~nm}) /$ copolymer/Si(100) surface and, second, there should be a strain induced effect due to the local variations in the mechanical properties of the copolymer template.
The magnetic properties of the $\mathrm{Nd}-\mathrm{Co} /$ template samples have been characterized at room temperature by magnetooptical transverse Kerr effect (MOTKE): ${ }^{32}$ the incident light beam was linearly polarized parallel to the plane of incidence and a magnetic field $(\mathrm{H})$ was applied parallel to the sample plane and transverse to the optical plane of incidence. The reflected light was detected with a photodiode and the changes in reflectivity were recorded as a function of $\mathrm{H}$ in order to obtain the in-plane magnetization hysteresis loops. ${ }^{32}$ Low temperature extraordinary Hall effect (EHE) has been measured in a He cryostat with a $90 \mathrm{kOe}$ electromagnet in order to obtain the out-of-plane hysteresis loops of the PMA samples. ${ }^{33,34}$ Magnetic domain structure has been characterized at room temperature by magnetic force microscopy (MFM) using a Nanotec ${ }^{\mathrm{TM}}$ system equipped with a $1 \mathrm{kOe}$ electromagnet that allows us to apply in-plane variable fields. ${ }^{35}$

\section{RESULTS AND DISCUSSION}

Figure 3 shows the MOTKE in-plane hysteresis loops of a $52 \mathrm{~nm} \mathrm{Nd-Co/template} \mathrm{sample} \mathrm{with} \mathrm{the} \mathrm{magnetic} \mathrm{field}$ applied either along the in-plane easy axis (Fig. 3(a)) or along the in-plane hard axis (Fig. 3(b)), in comparison with the data for a $52 \mathrm{~nm}$ Nd-Co film deposited on a flat Si substrate. All the loops display the typical "transcritical" shape characteristic of materials with weak perpendicular magnetic anisotropy: ${ }^{20,36}$ a reduced in-plane remanent magnetization $\left(\mathrm{M}_{\mathrm{R}}\right)$ followed by an almost linear reversible region as the magnetization approaches saturation. This behaviour is typical of low-Q materials and is caused by the nucleation of weak stripe domains with an alternating out-of-plane magnetization component and a mean in-plane magnetization component along the stripe direction. ${ }^{20,21}$ Stripe domains tend to align along the direction of the last saturating field resulting in an almost isotropic in-plane magnetic behaviour, that is, magnetic films with stripe domains display the so called in-plane "rotatable" anisotropy. ${ }^{37,38}$ This is indeed the case for the loops displayed in Fig. 3: for the $\mathrm{Nd}-\mathrm{Co} / \mathrm{template}$ film, the easy and hard axis remanences are very similar $\left(\mathrm{M}_{\mathrm{R}}^{\mathrm{EA}} / \mathrm{M}_{\mathrm{S}}=0.52\right.$ and $\left.\mathrm{M}_{\mathrm{R}}^{\mathrm{HA}} / \mathrm{M}_{\mathrm{S}}=0.42\right)$ and, also, the coercivity $\left(\mathrm{H}_{\mathrm{C}}\right)$ is almost independent
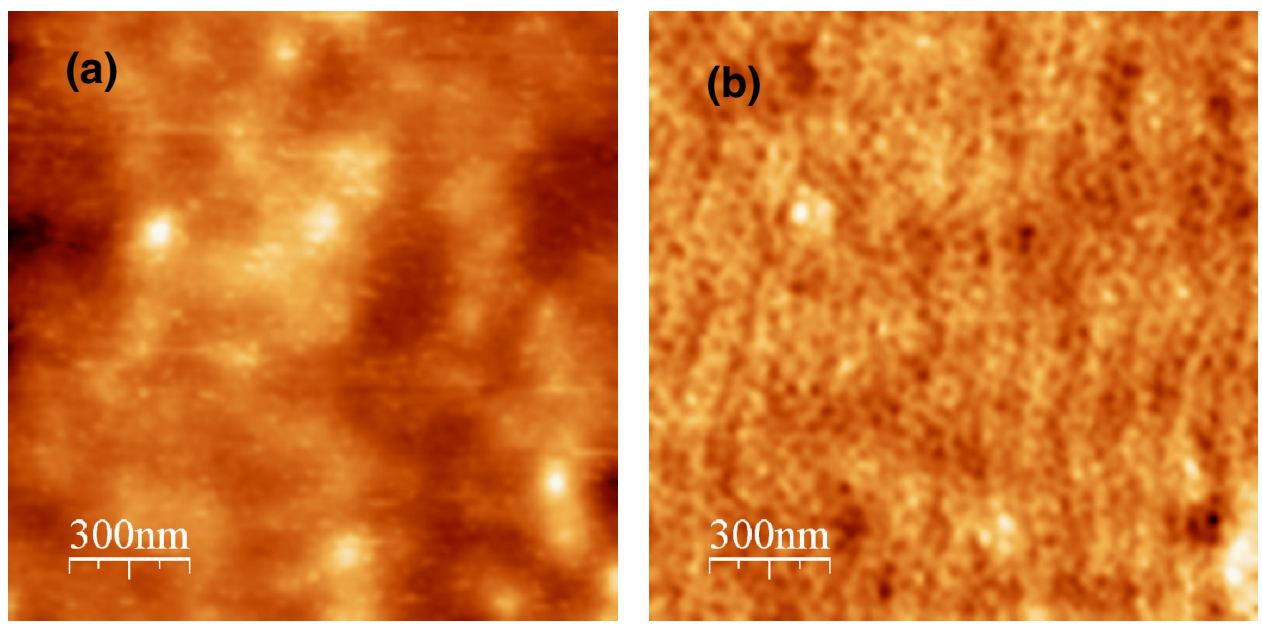

FIG. 2. AFM images of $45 \mathrm{~nm} \mathrm{Nd-Co/}$ template sample: (a) height image (b) phase image. 

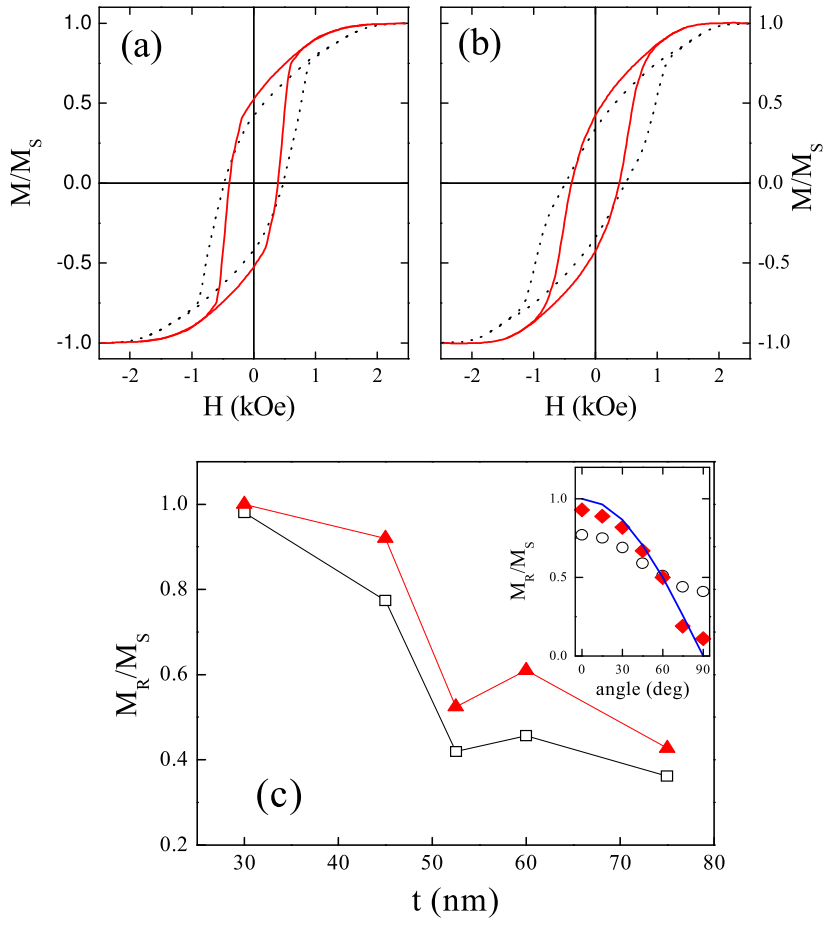

FIG. 3. MOTKE in-plane hysteresis loops of $52 \mathrm{~nm}$ Nd-Co films with (a) $\mathrm{H}$ along in-plane easy axis and (b) $\mathrm{H}$ along in plane hard axis: solid line, $\mathrm{Nd}-$ Co/template; dotted line, $\mathrm{Nd}-\mathrm{Co} / \mathrm{Si}(100)$. (c) Thickness dependence of easy axis remanent magnetization of $\mathrm{NdCo}$ films: squares, $\mathrm{Nd}-\mathrm{Co} / \mathrm{Si}$; triangles, $\mathrm{NdCo} /$ template. Inset shows the in-plane angular dependence of $\mathrm{M}_{R} / \mathrm{M}_{\mathrm{S}}$ for $45 \mathrm{~nm} \mathrm{NdCo} / \mathrm{Si}$ (circles) and $45 \mathrm{~nm} \mathrm{Nd-Co/template} \mathrm{(rhombs).} \mathrm{Angles} \mathrm{are}$ measured from the in-plane easy axis. Solid line corresponds to the behavior of in-plane uniaxial anisotropy $\mathrm{M}_{\mathrm{R}} / \mathrm{M}_{\mathrm{S}}=\cos \theta$.

of the in-plane direction of the magnetic field with easy axis $\mathrm{H}_{\mathrm{C}}{ }^{\mathrm{EA}}=390 \mathrm{Oe}$ and hard axis $\mathrm{H}_{\mathrm{C}}{ }^{\mathrm{HA}}=380 \mathrm{Oe}$. Something similar happens for the $\mathrm{Nd}-\mathrm{Co} / \mathrm{Si}$ film: the remanent magnetization and the coercivity do not change much from the in-plane easy axis $\left(\mathrm{M}_{\mathrm{R}}{ }^{\mathrm{EA}} / \mathrm{M}_{\mathrm{S}}=0.43\right.$ and $\left.\mathrm{H}_{\mathrm{C}}{ }^{\mathrm{EA}}=480 \mathrm{Oe}\right)$ to the in-plane hard axis $\left(\mathrm{M}_{\mathrm{R}}{ }^{\mathrm{HA}} / \mathrm{M}_{\mathrm{S}}=0.34\right.$ and $\left.\mathrm{H}_{\mathrm{C}}{ }^{\mathrm{HA}}=490 \mathrm{Oe}\right)$. The comparison between the hysteresis loops of these two $52 \mathrm{~nm}$ thick Nd-Co samples shows that the main effect of the copolymer template is to reduce the in-plane saturation field and to increase the remanent magnetization of the sample grown on the copolymer template in comparison with the one deposited on the flat $\mathrm{Si}$ substrate.

The same qualitative trend is observed in the whole thickness range studied. Figure 3(c) shows the evolution the in-plane easy axis remanence $\mathrm{M}_{\mathrm{R}}{ }^{\mathrm{EA}} / \mathrm{M}_{\mathrm{S}}$ upon increasing magnetic layer thickness $t$ for both series of Nd-Co films. For $\mathrm{t}=30 \mathrm{~nm}, \mathrm{M}_{\mathrm{R}}{ }^{\mathrm{EA}} / \mathrm{M}_{\mathrm{S}}$ is close to unity in both cases. This indicates that the magnetization is still within the sample plane for this small thickness. As $\mathrm{t}$ becomes larger, $\mathrm{M}_{\mathrm{R}}{ }^{\mathrm{EA}} / \mathrm{M}_{\mathrm{S}}$ decreases down to $\mathrm{M}_{\mathrm{R}}{ }^{\mathrm{EA}} / \mathrm{M}_{\mathrm{S}} \approx 0.4$ for $\mathrm{t}=75 \mathrm{~nm}$ indicating the gradual out-of-plane magnetization rotation induced by perpendicular magnetic anisotropy as the samples become thicker and the magnetostatic energy term decreases. This behavior is typical of materials with weak perpendicular magnetic anisotropy: $\mathrm{M}$ lies in plane for the thinnest magnetic layers until stripe domains are nucleated above a certain critical thickness that depends on material parameters. ${ }^{20,21}$ In all the cases, the $\mathrm{M}_{\mathrm{R}}{ }^{\mathrm{EA}} / \mathrm{M}_{\mathrm{S}}$ curve of the $\mathrm{Nd}-\mathrm{Co} /$ template samples lies above the data from the $\mathrm{Nd}-\mathrm{Co} / \mathrm{Si}(100)$ films, indicating a lower perpendicular magnetic anisotropy in the nanostructured samples.

The transition between in-plane and out-of-plane anisotropy regimes occurs close to $\mathrm{t}=45 \mathrm{~nm}$, which is also the point at which the differences between both kinds of samples are more significant. This can be seen, for example, in the angular dependence of the remanent in-plane magnetization shown in the inset of Fig. 3: for the $45 \mathrm{~nm} \mathrm{Nd-Co/template}$ sample, $\mathrm{M}_{\mathrm{R}}(\theta)$ can be reasonably described by a $\cos (\theta)$ law characteristic of in-plane uniaxial anisotropy whereas the data of the $45 \mathrm{~nm} \mathrm{Nd-Co/Si} \mathrm{film} \mathrm{follow} \mathrm{a} \mathrm{more} \mathrm{isotropic}$ trend indicating the onset of "rotatable" anisotropy. A clearer picture of the domain structure and magnetization reversal of
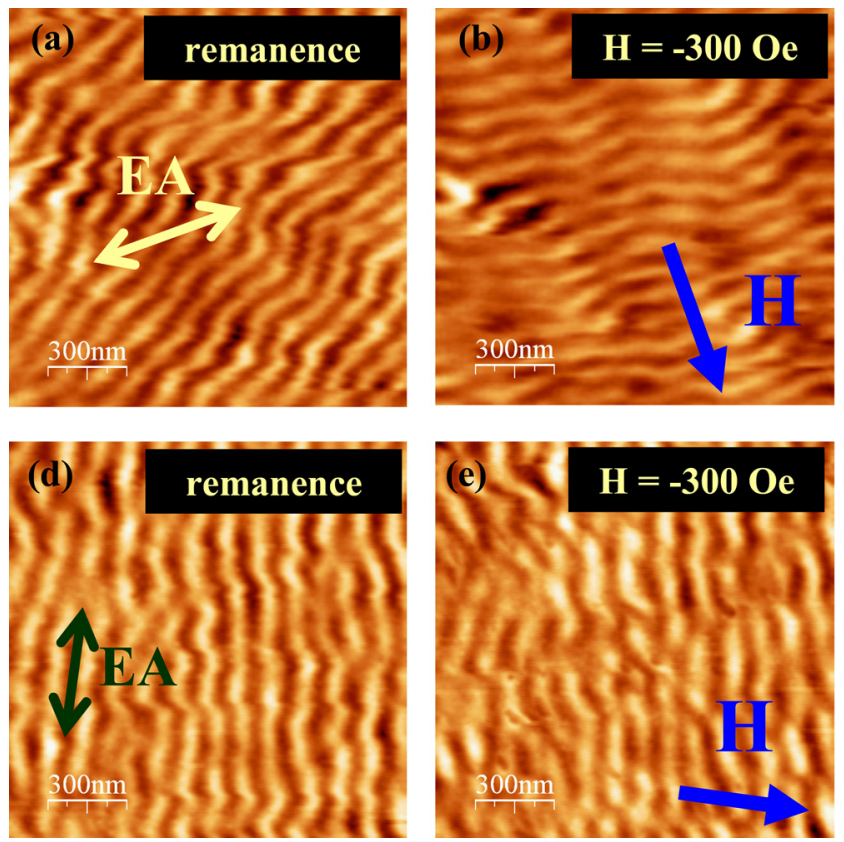

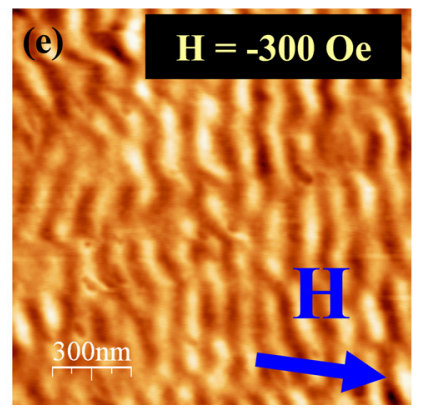

FIG. 4. MFM images during a hard axis magnetization process: (a)-(c) $45 \mathrm{~nm}$ $\mathrm{Nd}-\mathrm{Co} / \mathrm{Si}(100)$ and (d)-(f) $45 \mathrm{~nm} \mathrm{Nd-Co/}$ template. Arrows indicate applied field direction and in-plane easy axis.

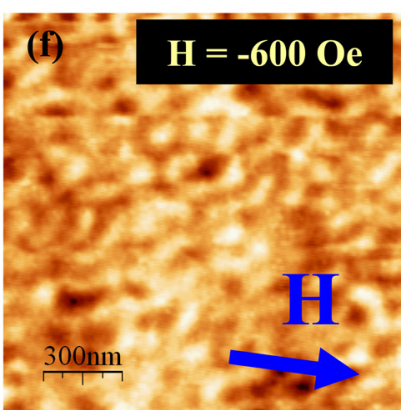


these samples can be obtained by field dependent magnetic force microscopy. Figure 4(a) is a MFM image of the remanent magnetization state of the $45 \mathrm{~nm} \mathrm{Nd-Co/Si} \mathrm{film} \mathrm{after}$ saturating along the in-plane hard axis direction. A well defined stripe domain structure can be observed with period $\Lambda=115 \mathrm{~nm}$. However, the stripes are neither aligned with the in-plane easy axis nor with the direction of the last applied field (i.e., perpendicular to the easy axis). Rather, they lie halfway between the field and the in-plane easy axis direction, in agreement with the non-zero hard axis remanence observed in Fig. $3\left(\mathrm{M}_{\mathrm{R}}\left(90^{\circ}\right) / \mathrm{M}_{\mathrm{S}}=0.41\right)$. Then, as a reversed hard axis field of increasing magnitude is applied to the sample, the stripes rotate towards the applied field in a continuous fashion (Figs. 4(b) and 4(c)). The stripe pattern reaches the applied field direction around 750 Oe (Fig. 4(c)) which is close to the in-plane hard axis saturation field obtained in the hysteresis loops $\left(\mathrm{H}_{\mathrm{S}}{ }^{\mathrm{HA}}=820 \mathrm{Oe}\right)$. A different behaviour is found in the $\mathrm{Nd}-\mathrm{Co} /$ template sample: the remanent state is characterized by a weak stripe domain pattern of period $\Lambda=125 \mathrm{~nm}$ aligned along the easy axis direction, irrespective of the direction of the last saturating field (see Fig. 4(d)). Then, upon increasing the hard axis field magnitude, stripes do not rotate (Fig. 4(e)) but, rather, they begin to loose their continuity until they decay into an irregular bubble pattern (Fig. 4(f)) for fields close to the in-plane hard axis saturation field $\left(\mathrm{H}_{\mathrm{S}}{ }^{\mathrm{HA}}=650 \mathrm{Oe}\right)$. These results can be understood in terms of the competition between in-plane uniaxial anisotropy, induced by growth conditions and "rotatable" anisotropy associated to the stripe domain configuration. The in-plane uniaxial anisotropy field $\left(\mathrm{H}_{\mathrm{K}}{ }^{\prime \mid}\right)$ of these $45 \mathrm{~nm}$ Nd-Co films is $\mathrm{H}_{\mathrm{K}}{ }^{\|}=380 \mathrm{Oe}$, calculated from the slope of the hard axis loop. On the other hand, "rotatable" anisotropy produces a "pseudouniaxial" anisotropy field $\left(\mathrm{H}_{\mathrm{K}}{ }^{\text {rot }}\right)$ that is given ${ }^{38}$ by

$$
\mathrm{H}_{\mathrm{K}}{ }^{\mathrm{rot}}=8 \mathrm{M}_{\mathrm{S}} \mathrm{J}_{2}^{2}(\alpha) / \mathrm{J}_{0}(\alpha)(\pi-\Lambda / 4 \mathrm{t}[1-\exp (-4 \pi \mathrm{t} / \Lambda)]),
$$

where $J_{\mathrm{n}}$ are the Bessel functions of the first kind and order n. $\alpha$ is the angular amplitude of the out of plane magnetization oscillation within the stripe pattern and can be determined from the average in-plane magnetization component along the stripes direction as $\left\langle M_{\text {in_plane }}\right\rangle=M_{S} J_{0}(\alpha)$. Thus, taking into account that in the easy axis loops the stripe pattern is along the field direction for both samples, we can use the $\mathrm{M}_{\mathrm{R}}{ }^{\mathrm{EA}}$ values and the measured stripe periods to estimate the rotatable anisotropy field as $\mathrm{H}_{\mathrm{K}}{ }^{\text {rot }}(45 \mathrm{~nm} \mathrm{Nd-Co/tem-}$ plate $) \approx 30 \mathrm{Oe}$ and $\mathrm{H}_{\mathrm{K}}{ }^{\text {rot }}(45 \mathrm{~nm} \mathrm{Nd}-\mathrm{Co} / \mathrm{Si}) \approx 300 \mathrm{Oe}$. In the first case, $\mathrm{H}_{\mathrm{K}}{ }^{\text {rot }}$ is only a small perturbation to $\mathrm{H}_{\mathrm{K}}{ }^{\|}$, and the sample behaviour is dominated by in-plane uniaxial anisotropy so that the magnetization always rotates back to the easy axis in the remanent state. On the other hand, the stronger perpendicular magnetic anisotropy of the $\mathrm{Nd}-\mathrm{Co}$ film deposited on the $\mathrm{Si}$ substrate results in a larger $\mathrm{H}_{\mathrm{K}}{ }^{\text {rot }}(45 \mathrm{~nm}$ $\mathrm{Nd}-\mathrm{Co} / \mathrm{Si}$ ) that becomes comparable to the growth induced in-plane uniaxial anisotropy. This results in the more isotropic in-plane $\mathrm{M}_{\mathrm{R}}(\theta)$ curve and the mixed behaviour observed during the MFM characterization.

Now, it is interesting to compare the magnetic scale set by the stripe pattern period $(\Lambda=115-125 \mathrm{~nm}$, as seen in Fig. 4) with the structural disorder scale (about $50 \mathrm{~nm}$, as seen in Fig. 2), which is much smaller than in previous works with irradiated samples. ${ }^{4,6}$ In our case, magnetic domains are larger than the locally modified regions grown on the copolymer pores. Thus, interaction between magnetic domains and structural defects should not occur on a one-to-one basis but, rather, it should show up as a global enhancement of domain pinning effects. This is actually observed in the out-of-plane magnetic EHE hysteresis loops shown in Fig. 5 for two 45 $\mathrm{nm} \mathrm{Nd-Co} \mathrm{films} \mathrm{grown} \mathrm{either} \mathrm{on} \mathrm{Si}(100)$ (Fig. 5(a)) or on the nanostructured template (Fig. 5(b)). In both cases, the loops display the typical shape of weak perpendicular anisotropy films with a large high field hysteresis and a thinner low field "waist": ${ }^{39-41}$ as the out-of-plane field is reduced from saturation, reversed domains begin to nucleate at a positive field $\mathrm{H}_{\mathrm{N}}\left(\mathrm{H}_{\mathrm{N}} \approx 3.5 \mathrm{kOe}\right.$ for Nd-Co/template and $4 \mathrm{kOe}$ for $\mathrm{Nd}-$ $\mathrm{Co} / \mathrm{Si}$ ). Then, magnetization reversal proceeds by a propagation process, until the magnetization is fully reversed by the annihilation of the last nuclei with the initial magnetization orientation at a larger negative field $\mathrm{H}_{\mathrm{ann}}\left(\mathrm{H}_{\mathrm{ann}} \approx-7.6 \mathrm{kOe}\right.$ for $\mathrm{Nd}-\mathrm{Co} /$ template and $-8.2 \mathrm{kOe}$ for $\mathrm{Nd}-\mathrm{Co} / \mathrm{Si}$ ). The main differences between both samples are twofold: first, the characteristic field values are lower for the nanostructured sample, which is in agreement with the lower effective perpendicular magnetic anisotropy observed in the in-plane magnetic characterization; second, the hysteresis at low

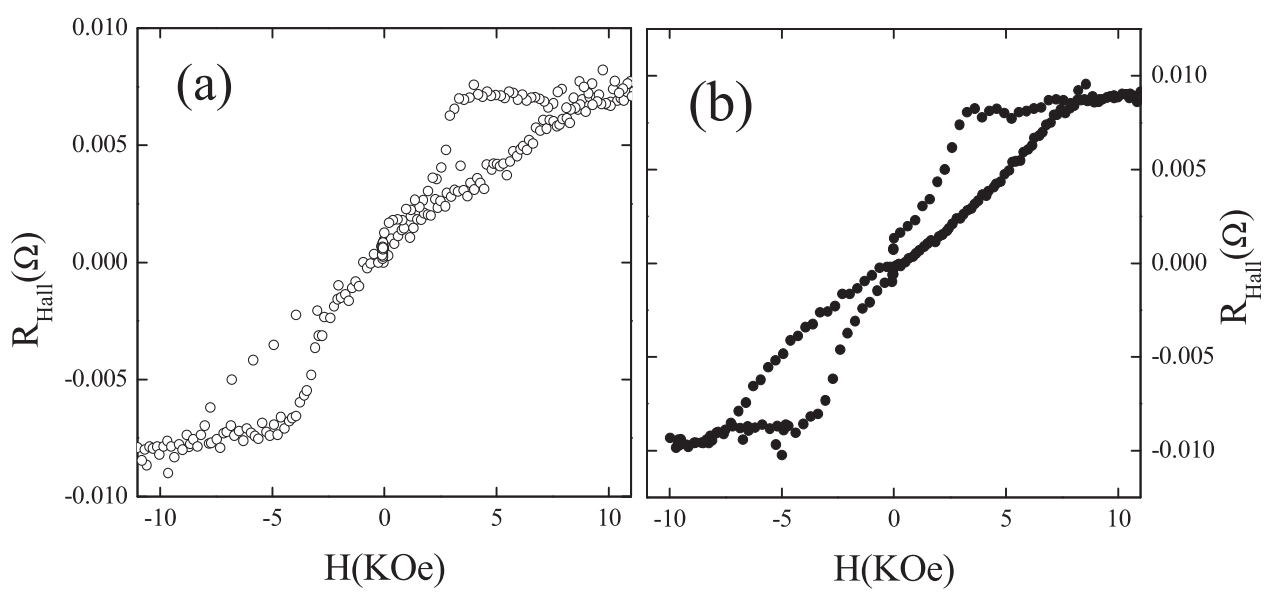

FIG. 5. EHE out-of-plane hysteresis loops measured at $10 \mathrm{~K}$ : (a) $45 \mathrm{~nm} \mathrm{Nd}$ $\mathrm{Co} / \mathrm{Si}(100)$, (b) $45 \mathrm{~nm} \mathrm{Nd}-\mathrm{Co} /$ template. 
fields is much larger for the Nd-Co/template film. Actually, the out-of-plane hysteresis loop of the $\mathrm{Nd}-\mathrm{Co} / \mathrm{Si}$ sample displays a $4 \mathrm{kOe}$ wide reversible region around remanence that is absent in the hysteresis loop of the nanostructured sample. Thus, low field domain propagation is hindered in the Nd$\mathrm{Co} /$ template sample indicating the larger number of domain pinning centres, associated with the structural disorder induced by the copolymer template.

\section{CONCLUSION}

Self-organized (PS-b-P4VP) copolymer layers have been fabricated as templates for the deposition of perpendicular magnetic anisotropy amorphous Nd-Co films. The topography of the di-block copolymer layers consists of $60 \mathrm{~nm}$ spaced pores arranged in a hexagonal geometry that is conserved after the deposition of a $10 \mathrm{~nm}$ Al buffer layer. These nanostructured $\mathrm{Al} /$ copolymer templates have a two fold influence on the magnetic films grown on top: a topographic effect due to the corrugated surface geometry and a strain effect due to the local variations of the copolymer mechanical properties. The effect of nanostructuring on the magnetic properties of the Nd-Co films is particularly important in the thickness range corresponding to the transition between inplane and out-of-plane anisotropy regimes: the Nd-Co/template samples present lower effective PMA in comparison with reference $\mathrm{Nd}-\mathrm{Co} / \mathrm{Si}(100)$ films and enhanced irreversibility of the out-of-plane hysteresis loops due to domain pinning effects.

\section{ACKNOWLEDGMENTS}

Work supported by Spanish MEC under Grants FIS200806249 and Consolider CSD2007-00010 and by CAM under Grant S2009/MAT-1726. We acknowledge helpful discussions with Professor T. P. Russell and Dr. R. Morales.

${ }^{1}$ J. A. Fernandez-Baca and W.-Y. Ching, The Magnetism of Amorphous Metals and Alloys (World Scientific, Singapore, 1995).

${ }^{2}$ M. Mansuripur, The Physical Principles of Magnetooptical Recording (Cambridge University Press, Cambridge, 1995).

${ }^{3}$ D. Ravelosona, T. Devolder, C. Chappert, H. Bernas, Y. Chen, J. P. Jamet, J. Ferré, E. Cambril, and V. Mathet, Mater. Sci. Eng. 15, 53 (2001).

${ }^{4}$ D.G. Merkel, L. Bottyan, F. Tancziko, Z. Zolnai, N. Nagy, G. Vertesy, J. Waizinger, and L. Bommer, J. Appl. Phys. 109, 124302 (2011).

${ }^{5}$ A. Aziz, S. J. Bending, H. Roberts, S. Crampin, P. J. Heard, and C. H. Marrows, J. Appl. Phys. 98, 124102 (2005).

${ }^{6}$ S. Konings, J. Miguel, J. Goedkoop, J. Camarero, and J. Vogel, J. Appl. Phys. 100, 033904 (2006).

${ }^{7}$ O. Hellwig, A. Moser, E. Dobisz, Z. Z. Bandic, H. Yang, D. S. Kercher, J. Risner, J. D. Amtgaard, D. Yaney, and E. E. Fullerton, Appl. Phys. Lett. 93, 192501 (2008).
${ }^{8}$ Y. J. Oh, C. A. Ross, Y. Wang, and C. V. Thompson, Small 5, 860 (2009).

${ }^{9}$ C. A. Ross and J. Y. Cheng, MRS Bull. 33, 838 (2008).

${ }^{10}$ J. Bang, U. Jeong, D. Y. Ryu, T. P. Russell, and C. J. Hawker, Adv. Mater. 21, 4769 (2009).

${ }^{11}$ S. Park and T. P. Russell, Nano 5, 1 (2010).

${ }^{12}$ B. D. Terris and T. Thomson, J. Phys. D: Appl. Phys. 38, R199 (2005)

${ }^{13}$ S. B. Darling and S. D. Bader, J. Mater. Chem. 15, 4189 (2005).

${ }^{14}$ M. T. Rahman, N. N. Shams, C. H. Lai, J. Fidler, and D. Suess, Phys. Rev. B 81, 014418 (2010).

${ }^{15}$ M. T. Rahman, X. Liu, and A. Morisako, J. Appl. Phys. 99, 08G904 (2006).

${ }^{16}$ D. Navas, F. Ilievski, and C. A. Ross, J. Appl. Phys. 105, 113921 (2009).

${ }^{17}$ K. Liu, S. M. Baker, M. Tuominen, T. P. Russell, and I. K. Schuller, Phys. Rev. B 63, 060403(R) (2001).

${ }^{18}$ A. Baruth, M. D. Rodwogin, A. Shankar, M. J. Erickson, M. A. Hillmyer, and C. Leighton, ACS Appl. Mater. Interfaces 3, 3472 (2011).

${ }^{19}$ V. P. Chuang, W. Jung, C. A. Ross, J. Y. Cheng, O.-H. Park, and H.-C. Kim, J. Appl. Phys. 103, 074307 (2008).

${ }^{20}$ A. Hubert and R. Schafer, Magnetic Domains: The Analysis of Magnetic Microstructures (Springer, Berlin, 1998).

${ }^{21}$ Y. Murayama, J. Phys. Soc. Jpn. 21, 2253 (1966).

${ }^{22}$ R. Cid, G. Rodriguez-Rodriguez, L. M. Alvarez-Prado, J. Diaz, and J. M. Alameda, J. Magn. Magn. Mater. 316, e446 (2007).

${ }^{23}$ R. C. Taylor, J. R. McGuire, J. M. D. Coey, and A. Gangulee, J. Appl. Phys. 49, 2885 (1978).

${ }^{24}$ Y. Suzuki, S. Takayama, F. Kirino, and N. Ohta, IEEE Trans. Magn. 23, 2275 (1987).

${ }^{25}$ R. Cid, J. Díaz, L. M. Álvarez-Prado, J. M. Alameda, S. M. Valvidares, J. C. Cezar, and N. B. Brookes, J. Phys.: Conf. Ser. 200, 072017 (2010).

${ }^{26}$ S. Park, O. Yavuzcetin, B. Kim, M. T. Tuominen and T. P. Russell, Small 5, 1064 (2009).

${ }^{27}$ S. Park, J.-Y. Wang, B. Kim, W. Chen, and T. P. Russell, Macromolecules 40, 9059 (2007).

${ }^{28}$ M. M. Abul Kashem, G. Kaune, A. Diethert, W. Wang, K. Schlage, S. Couet, R. Rölsberger, S. V. Roth, and P. Müller-Buschbaum, Macromolecules 44, 1621 (2011).

${ }^{29}$ J. Tamayo and R. García, Langmuir 12, 4430 (1996); Appl. Phys. Lett. 73, 2926 (1998)

${ }^{30}$ M. J. Fasolka, A. M. Mayes, and S. N. Magonov, Ultramicroscopy 90, 21 (2001).

${ }^{31}$ B. Bhushan and J. Qi, Nanotechnology 14, 886 (2003).

${ }^{32}$ C. Dehesa-Martínez, L. Blanco-Gutierrez, M. Vélez, J. Díaz, L. M. Alvarez-Prado, and J. M. Alameda, Phys. Rev. B 64, 024417 (2001).

${ }^{33}$ C. L. Canedy, X. W. Li, and G. Xiao, Phys. Rev. B 62, 508 (2000).

${ }^{34}$ G. Rodriguez-Rodriguez, J. L. Menéndez, A. Perez-Junquera, A. HierroRodriguez, N. Montenegro, D. Ravelosona, J. M. Alameda, and M. Velez, J. Phys.: Conf. Ser. 200, 042021 (2009).

${ }^{35}$ F. Valdés-Bango, A. Hierro-Rodriguez, J. M. Teixeira, G. N. Kakazei, Y. G. Pogorelov, G. Rodriguez-Rodriguez, M. Vélez, J. M. Alameda, J. B. Sousa, and J. I. Martin, J. Nanosci. Nanotechnol. 12, 7510 (2012).

${ }^{36}$ D. H. Shin, G. Suran, D. Chumakov, R. Schafer, and Y. Henry, J. Appl. Phys. 91, 8237 (2002).

${ }^{37}$ M. S. Cohen, J. Appl. Phys. 33, 2968 (1962); N. Saito, H. Fujiwara, and Y. Suzita, J. Phys. Soc. Jpn. 19, 1116 (1964).

${ }^{38}$ L. M. Alvarez-Prado, G. T. Perez, R. Morales, F. H. Salas, and J. M. Alameda, Phys. Rev. B 56, 3306 (1997).

${ }^{39}$ M. Hehn, S. Padovani, K. Ounadjela, and J. P. Bucher, Phys. Rev. B 54, 3428 (1996)

${ }^{40}$ O. Hellwig, G. P. Denbeaux, J. B. Kortright, and E. E. Fullerton, Physica B 336, 136 (2003).

${ }^{41}$ E. A. Jagla, Phys. Rev. B 72, 094406 (2005). 